\title{
5. Introduction
}

\section{J.F. Feenstra and P.E.M. Lammers}

\section{THE CLIMATE CHANGE POLICY FIELD}

Naturally occurring gases (water vapour, carbon dioxide, methane, nitrous oxide and ozone) in the earth's atmosphere create a (natural) greenhouse effect on the earth, resulting in the earth's climate as we know it. Increase of the concentrations of these gases results in an enhanced greenhouse effect and thereby changes the earth's climate.

Since the beginning of the Industrial Revolution the atmospheric concentrations of most of the greenhouse gases have increased dramatically and are expected to increase even more in the decades to come. The largest emission sources of carbon dioxide, methane and nitrous oxide are the combustion of fossil fuels, deforestation and agriculture. Expected impacts of (future) climate change include those on agriculture and food security, coastal zones due to sea-level rise, ecosystems and human health, and water resources and human infrastructure. Policy-makers have started to acknowledge the necessity of adaptation to climate change impacts.

The chapters in this part of the volume provide an overview of the main issues related to climate change. Each has been written by a recognized expert (see Introduction to the contributors).

\section{RELATIONS TO OTHER POLICY FIELDS}

The Climate Change policy field is closely related to the policy fields of Ozone Layer Depletion, Air Pollution, Urban Environmental Problems and Loss of Biodiversity. The chapter by Grassl specifically highlights these relationships.

The relation with the Ozone Layer Depletion policy field is the most direct of those mentioned above. Ozone is a naturally occurring greenhouse gas, and therefore a decrease of the stratospheric ozone concentration will result in radiative cooling. However, the ozone-depleting substances (for example 
CFCs and halons) are effective greenhouse gases which enhance the greenhouse effect.

The relationship with the Air Pollution policy field is important due to the cooling effect of aerosols. Emissions of sulphur dioxide and soot (causing acid rain) result in atmospheric aerosols that reduce the incoming solar radiation and thereby have a cooling effect. In contrast to the global warming effect of greenhouse gases, the cooling effect of aerosols is regional. Reduction of emissions of these aerosols will result in a decrease in this regional cooling effect and make the warming of the atmosphere more apparent in highly industrialized regions. This means that an improvement in the environmental situation with regard to acid rain and regional air pollution results in a worse situation as far as global warming/climate change is concerned. In practice, it is hard to identify how climate has been changed at regional level by the opposing effects of aerosols and greenhouse gases.

Another link between the Climate Change and Air Pollution policy fields is related to the measures to reduce emissions of air pollutants. Measures taken to reduce carbon dioxide emissions will influence the emissions of other air pollutants as well. Therefore emission reduction measures should be focused on these win-win situations.

Since air pollution is a major component of urban problems, the above relation with the Air Pollution policy field means that there is also a relation between the Climate Change and Urban Environmental Problems policy fields.

A relation between the policy fields Climate Change and Loss of Biodiversity exists in the areas of deforestation, reforestation and afforestation. Deforestation is one of the main contributors to climate change. By decreasing the forest areas, the existing sinks of carbon dioxide are reduced. The burning of wood gives rise to emissions of greenhouse gases such as carbon dioxide, methane, nitrous oxide, nitrogen oxides. Reforestation and afforestation increase the sinks of carbon dioxide and have thereby a positive effect on climate change. Since tropical forests are the largest source of biodiversity, any changes in the area of tropical forests will have effects on biodiversity. Therefore, measures to protect biodiversity are likely to have a positive effect in the Climate Change policy field as well.

\section{INTERNATIONAL FRAMEWORK}

At the Earth Summit (Second UN Conference on Environment and Development) in Rio de Janeiro in June 1992, the United Nations Framework Convention on Climate Change (UNFCCC) was agreed and signed by 154 countries. The convention came into force on 21 May 1994 after 50 countries 
ratified the convention. The main objective of the UNFCCC is given in Article 2:

The ultimate objective of this convention ... is to achieve... stabilisation of greenhouse gas concentrations in the atmosphere at a level that would prevent dangerous anthropogenic interference with the climate system. Such a level should be achieved within a time frame sufficient to allow ecosystems to adapt naturally to climate change, to ensure that food production is not threatened and to enable economic development to proceed in a sustainable manner.

As Houghton states in his chapter, 'the best way to meet the demands of this objective will require a great deal of debate and discussion'. Grassl illustrates this point by mentioning the inability of the first Conference of Parties (CoP 1) to the Convention to agree on even a weak $\mathrm{CO}_{2}$ protocol and the subsequent postponement of such a protocol to the CoP in Kyoto in December 1997. Further intense debate surrounded the Kyoto Conference and the agreement reached was viewed by many as containing many loopholes.

\section{Research Activities}

Climate change research is carried out in numerous national and international programmes, including the Environment and Climate Programme of the European Union. Results of climate change studies are combined and assessed by the Intergovernmental Panel on Climate Change (IPCC). This panel, in which 1500 scientists from all over the world participate, was initiated by UNEP and WHO in 1988. The first scientific assessment of this intergovernmental panel was published in 1992 and can be seen as the precursor to the UNFCCC.

The Second Assessment report, published in 1996, was clearer on the impacts of human activities on climate. This report stated in the summary for policy-makers that: 'the balance of evidence suggests a discernible human influence on global climate'. This statement makes clear that human activities are already influencing global climate. Political measures are needed to prevent further influences, which could have irreversible effects.

\section{OVERVIEW OF PRESSURE INDICATORS FOR CLIMATE CHANGE}

Figure 5.1 provides an overview of the key indicators for the Climate Change policy field. The selection of the indicators and corresponding scores are the results of the second-round Eurostat Pressure Indicators Project expert questionnaire. The figure provides the ranking of each indicator for policy relevance, 
Pressure indicators for climate change: 'quality' questions Q1-3, core-ranked

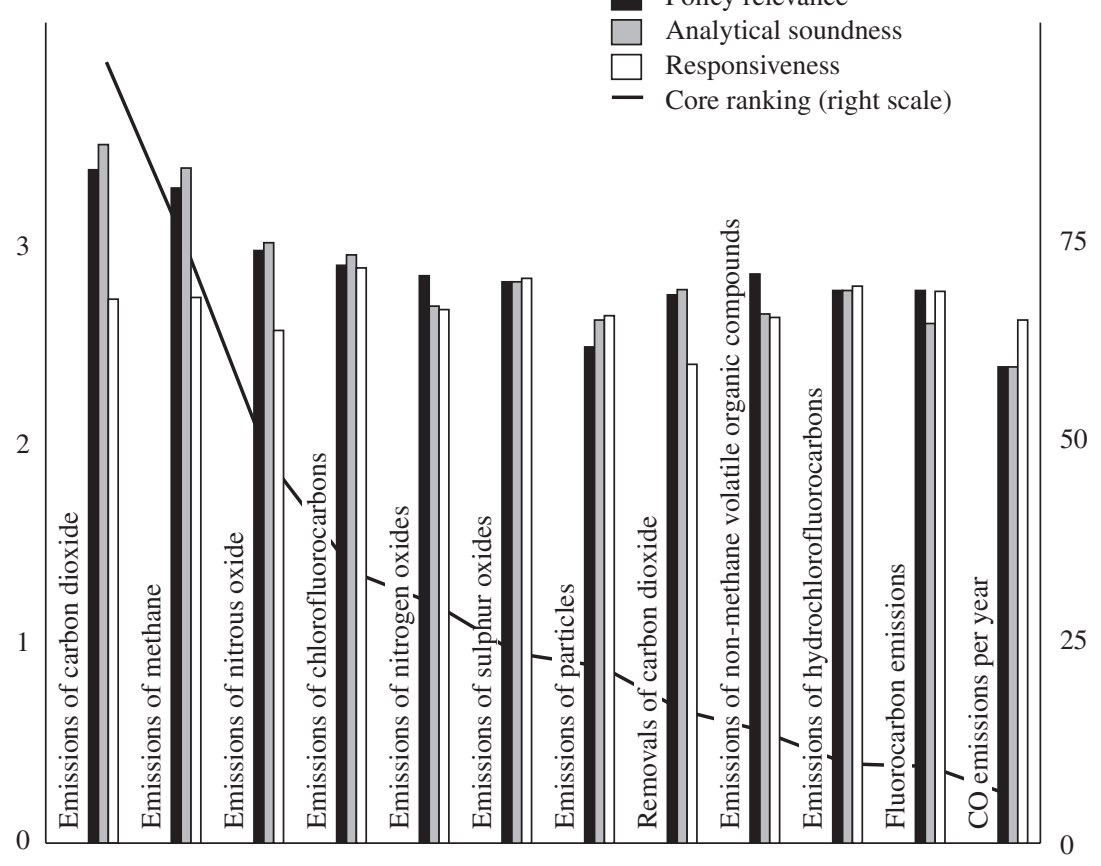

Ranking ( 1 = very low, 4 = very high):

Policy relevance Analytical soundness Responsiveness

Core ranking (right scale)

Figure 5.1 Results of second-round questionnaire for the Climate Change policy field

analytical soundness and responsiveness expressed as a score from 1 (very low) to 4 (very high). Also, the core ranking of the indicators is presented, expressed as a percentage of experts who included the indicator in their top five list of essential indicators.

The indicators for the direct greenhouse gases $\mathrm{CO}_{2}, \mathrm{CH}_{4}$ and $\mathrm{N}_{2} \mathrm{O}$ score highest for policy relevance, analytical soundness and core ranking. However, for responsiveness they score lower (although differences are not large). This indicates that emissions of these direct greenhouse gases are believed to be more difficult to reduce than emissions of halocarbons or sulphur dioxides.

The indicator for CFC emissions scores fourth, except for responsiveness where it scores first. This can easily be explained by the fact that the Montreal Protocol calls for a complete phasing out of CFC emissions. However, since this target has led to a drastic reduction of CFC emissions, it can be argued that CFCs should not score high in the set of top five core indicators. 
The detailed discussion on this issue in Part V on Ozone Layer Depletion is also applicable to the Climate Change policy field.

In section 6 of his chapter Grassl discusses the results of the second questionnaire. In his opinion, the indicators for $\mathrm{N}_{2} \mathrm{O}$ and $\mathrm{CFC}$ emissions are highly overrated and should not play a role in an integrated climate change index. In the case of $\mathrm{N}_{2} \mathrm{O}$, there is a lack of knowledge about the sources of emissions and, moreover, only limited possibilities exist to reduce emissions. In the case of CFCs, Grassl agrees with the point made above that since the most important CFCs are already phased out in the EU, they are not relevant to the list of climate change indicators.

Although Grassl is correct on the limited possibilities of reducing $\mathrm{N}_{2} \mathrm{O}$ emissions, this argument alone is not a sufficient reason to remove the $\mathrm{N}_{2} \mathrm{O}$ emission indicator from the set of climate change indicators. In international research and policy-making, $\mathrm{N}_{2} \mathrm{O}$ (together with $\mathrm{CO}_{2}$ and $\mathrm{CH}_{4}$ ) is considered to be one of the major greenhouse gases. This can be illustrated by the fact that, at the minimum, emissions of $\mathrm{CO}_{2}, \mathrm{CH}_{4}$ and $\mathrm{N}_{2} \mathrm{O}$ should be included in the national emission inventory as part of a National Communication. ${ }^{1}$ As shown in the scores on responsiveness, the European experts agree with Grassl that $\mathrm{N}_{2} \mathrm{O}$ emissions will be hard to reduce. Nevertheless, they rank the indicator in third place as a core indicator.

According to Grassl, the indicator for $\mathrm{NO}_{\mathrm{x}}$ emissions should score higher than in the results of the second questionnaire. His reasoning is that $\mathrm{NO}_{\mathrm{x}}$ contributes to many environmental problems and should therefore be considered more important. However, it should be noted that $\mathrm{NO}_{\mathrm{x}}$ is ranked highest as a core indicator in the Air Pollution policy field.

Houghton states that climate change indicators should relate to a wider context (sustainable development, other environmental problems). Indeed, some environmental pressures may play a role in several policy fields and have a large impact on total environmental quality, even though they may not be considered as a priority in any of the policy fields. If the effects in all policy fields were combined, the reduction of such a pressure might have a greater overall impact than the reduction of a priority pressure. This indicates an inherent disadvantage of dividing environmental pressures into different policy fields. The suggestion of valuing the importance of the indicator to other policy fields within the ranking of one particular policy field will lead to overvaluing the importance of the pressure indicator to that field.

The development of an additional set of core indicators to mirror the integrated environmental quality may be a solution to the points made by Grassl and Houghton. Based on the importance of a specific pressure indicator in each policy field where it is considered, an overall importance of this indicator for the environment as a whole could be estimated. This extra set of 
core indicators for the total environment could supplement the specific indicators for each policy field.

An issue not addressed in the chapters that follow is the policy relevance of the indicators for $\mathrm{SO}_{\mathrm{x}}$ and aerosol particles. Due to the regional cooling effect of these emissions, reductions will lead to a worsening of the climate change index. However, since emissions of $\mathrm{SO}_{\mathrm{x}}$ and aerosol particles have other negative environmental effects (acidification, human health), emission reductions are justified for reasons other than climate change. Therefore, the policy relevance of inclusion of these indicators into a climate change index is highly disputable.

\section{DISCUSSION AND CONCLUSIONS}

In general, the indicators for climate change are well defined and consensus between scientists is relatively high. However, there is discussion on the relative importance of indicators and the best set of core indicators. This relates to four particular indicators. First, $\mathrm{N}_{2} \mathrm{O}$, which is an important greenhouse gas, but whose emissions are hard to reduce; second, $\mathrm{NO}_{\mathrm{x}}$, which has a relatively limited importance for climate change, but may be a very important indicator for total environmental quality; third, CFCs, for which emissions have been reduced drastically due to the implementation of phasing-out actions under the Montreal Protocol, and finally, $\mathrm{SO}_{\mathrm{x}}$ and aerosol particles, for which the policy relevance of inclusion in a climate change index is highly disputable.

An issue not addressed in the chapters that follow is how to aggregate the indicators into one climate change index. Partly, the concept of global warming potentials, a measure of the relative radiative effects of greenhouse gases, can be used for this aggregation. However, global warming potentials have not been quantified for all greenhouse gases. The chapter on climate change in the TEPI publication on Indicator Definition ${ }^{2}$ provides a more detailed discussion on the various aspects of aggregation.

\section{NOTES}

1. A National Communication is a report on greenhouse gas emissions and mitigation policies and options that every party has to submit to the secretariat of the UNFCCC.

2. See Foreword. 